\title{
Precision Measurements of the Timelike Electromagnetic Form Factors of Pion, Kaon, and Proton
}

T. K. Pedlar, ${ }^{1}$ D. Cronin-Hennessy, ${ }^{2}$ K. Y. Gao, ${ }^{2}$ D. T. Gong, ${ }^{2}$ J. Hietala, ${ }^{2}$ Y. Kubota, ${ }^{2}$ T. Klein, ${ }^{2}$ B. W. Lang, ${ }^{2}$ S. Z. Li, ${ }^{2}$ R. Poling, ${ }^{2}$ A. W. Scott, ${ }^{2}$ A. Smith, ${ }^{2}$ S. Dobbs, ${ }^{3}$ Z. Metreveli, ${ }^{3}$ K. K. Seth, ${ }^{3}$ A. Tomaradze, ${ }^{3}$ P. Zweber, ${ }^{3}$ J. Ernst, ${ }^{4}$ K. Arms, ${ }^{5}$ H. Severini, ${ }^{6}$ S. A. Dytman, ${ }^{7}$ W. Love, ${ }^{7}$ S. Mehrabyan, ${ }^{7}$ J. A. Mueller, ${ }^{7}$ V. Savinov, ${ }^{7}$ Z. Li, ${ }^{8}$ A. Lopez,${ }^{8}$ H. Mendez,${ }^{8}$ J. Ramirez, ${ }^{8}$ G. S. Huang, ${ }^{9}$ D. H. Miller, ${ }^{9}$ V. Pavlunin, ${ }^{9}$ B. Sanghi, ${ }^{9}$ I. P. J. Shipsey, ${ }^{9}$ G. S. Adams,${ }^{10}$ M. Anderson, ${ }^{10}$ J.P. Cummings, ${ }^{10}$ I. Danko, ${ }^{10}$ J. Napolitano, ${ }^{10}$ Q. He, ${ }^{11}$ H. Muramatsu, ${ }^{11}$ C. S. Park, ${ }^{11}$ E. H. Thorndike, ${ }^{11}$ T. E. Coan, ${ }^{12}$ Y. S. Gao, ${ }^{12}$ F. Liu, ${ }^{12}$ M. Artuso, ${ }^{13}$ C. Boulahouache, ${ }^{13}$ S. Blusk, ${ }^{13}$ J. Butt, ${ }^{13}$ O. Dorjkhaidav, ${ }^{13}$ J. Li, ${ }^{13}$ N. Menaa, ${ }^{13}$ R. Mountain, ${ }^{13}$ K. Randrianarivony, ${ }^{13}$ R. Redjimi, ${ }^{13}$ R. Sia, ${ }^{13}$ T. Skwarnicki, ${ }^{13}$ S. Stone, ${ }^{13}$ J. C. Wang, ${ }^{13}$ K. Zhang, ${ }^{13}$ S. E. Csorna, ${ }^{14}$ G. Bonvicini,,${ }^{15}$ D. Cinabro, ${ }^{15}$ M. Dubrovin, ${ }^{15}$ A. Lincoln, ${ }^{15}$ A. Bornheim, ${ }^{16}$ S. P. Pappas, ${ }^{16}$ A. J. Weinstein, ${ }^{16}$ R. A. Briere,${ }^{17}$ G. P. Chen, ${ }^{17}$ J. Chen, ${ }^{17}$ T. Ferguson,,${ }^{17}$ G. Tatishvili,,${ }^{17}$ H. Vogel,,${ }^{17}$ M. E. Watkins,${ }^{17}$ J. L. Rosner, ${ }^{18}$ N.E. Adam, ${ }^{19}$ J.P. Alexander, ${ }^{19}$ K. Berkelman, ${ }^{19}$ D. G. Cassel,${ }^{19}$ J. E. Duboscq, ${ }^{19}$ K. M. Ecklund, ${ }^{19}$ R. Ehrlich, ${ }^{19}$ L. Fields, ${ }^{19}$ R. S. Galik, ${ }^{19}$ L. Gibbons, ${ }^{19}$ R. Gray, ${ }^{19}$ S. W. Gray,${ }^{19}$ D. L. Hartill,${ }^{19}$ B. K. Heltsley, ${ }^{19}$ D. Hertz,${ }^{19}$ C. D. Jones, ${ }^{19}$ J. Kandaswamy, ${ }^{19}$ D. L. Kreinick, ${ }^{19}$ V. E. Kuznetsov, ${ }^{19}$ H. Mahlke-Krüger, ${ }^{19}$ T. O. Meyer, ${ }^{19}$ P. U. E. Onyisi, ${ }^{19}$ J. R. Patterson, ${ }^{19}$ D. Peterson, ${ }^{19}$ E. A. Phillips, ${ }^{19}$ J. Pivarski, ${ }^{19}$ D. Riley, ${ }^{19}$ A. Ryd ${ }^{19}$ A. J. Sadoff, ${ }^{19}$ H. Schwarthoff, ${ }^{19}$ X. Shi, ${ }^{19}$ M. R. Shepherd, ${ }^{19}$ S. Stroiney, ${ }^{19}$ W. M. Sun, ${ }^{19}$ T. Wilksen, ${ }^{19}$ K. M. Weaver, ${ }^{19}$ M. Weinberger, ${ }^{19}$ S. B. Athar, ${ }^{20}$ P. Avery, ${ }^{20}$ L. Breva-Newell, ${ }^{20}$ R. Patel,,${ }^{20}$ V. Potlia,${ }^{20}$ H. Stoeck, ${ }^{20}$ J. Yelton, ${ }^{20}$ P. Rubin, ${ }^{21}$ C. Cawlfield,${ }^{22}$ B. I. Eisenstein, ${ }^{22}$ I. Karliner, ${ }^{22}$ D. Kim, ${ }^{22}$ N. Lowrey, ${ }^{22}$ P. Naik, ${ }^{22}$ C. Sedlack, ${ }^{22}$ M. Selen, ${ }^{22}$ E. J. White, ${ }^{22}$ J. Williams, ${ }^{22}$ J. Wiss, ${ }^{22}$ D. M. Asner, ${ }^{23}$ K. W. Edwards, ${ }^{23}$ and D. Besson ${ }^{24}$

\section{(CLEO Collaboration)}

\author{
${ }^{1}$ Luther College, Decorah, Iowa 52101, USA \\ ${ }^{2}$ University of Minnesota, Minneapolis, Minnesota 55455, USA \\ ${ }^{3}$ Northwestern University, Evanston, Illinois 60208, USA \\ ${ }^{4}$ State University of New York at Albany, Albany, New York 12222, USA \\ ${ }^{5}$ The Ohio State University, Columbus, Ohio 43210, USA \\ ${ }^{6}$ University of Oklahoma, Norman, Oklahoma 73019, USA \\ ${ }^{7}$ University of Pittsburgh, Pittsburgh, Pennsylvania 15260, USA \\ ${ }^{8}$ University of Puerto Rico, Mayaguez, Puerto Rico 00681 \\ ${ }^{9}$ Purdue University, West Lafayette, Indiana 47907, USA \\ ${ }^{10}$ Rensselaer Polytechnic Institute, Troy, New York 12180, USA \\ ${ }^{11}$ University of Rochester, Rochester, New York 14627, USA \\ ${ }^{12}$ Southern Methodist University, Dallas, Texas 75275, USA \\ ${ }^{13}$ Syracuse University, Syracuse, New York 13244, USA \\ ${ }^{14}$ Vanderbilt University, Nashville, Tennessee 37235, USA \\ ${ }^{15}$ Wayne State University, Detroit, Michigan 48202, USA \\ ${ }^{16}$ California Institute of Technology, Pasadena, California 91125, USA \\ ${ }^{17}$ Carnegie Mellon University, Pittsburgh, Pennsylvania 15213, USA \\ ${ }^{18}$ Enrico Fermi Institute, University of Chicago, Chicago, Illinois 60637, USA \\ ${ }^{19}$ Cornell University, Ithaca, New York 14853 \\ ${ }^{20}$ University of Florida, Gainesville, Florida 32611, USA \\ ${ }^{21}$ George Mason University, Fairfax, Virginia 22030, USA \\ ${ }^{22}$ University of Illinois, Urbana-Champaign, Illinois 61801, USA \\ ${ }^{23}$ Carleton University, Ottawa, Ontario, Canada K1S 5B6 and the Institute of Particle Physics, Canada \\ ${ }^{24}$ University of Kansas, Lawrence, Kansas 66045, USA \\ (Received 30 September 2005; published 21 December 2005)
}

Using $20.7 \mathrm{pb}^{-1}$ of $e^{+} e^{-}$annihilation data taken at $\sqrt{s}=3.671 \mathrm{GeV}$ with the CLEO-c detector, precision measurements of the electromagnetic form factors of the charged pion, charged kaon, and proton have been made for timelike momentum transfer of $\left|Q^{2}\right|=13.48 \mathrm{GeV}^{2}$ by the reaction $e^{+} e^{-} \rightarrow h^{+} h^{-}$. The measurements are the first ever with identified pions and kaons of $\left|Q^{2}\right|>4 \mathrm{GeV}^{2}$, with the results $F_{\pi}\left(13.48 \mathrm{GeV}^{2}\right)=0.075 \pm 0.008($ stat $) \pm 0.005$ (syst) $\quad$ and $\quad F_{K}\left(13.48 \mathrm{GeV}^{2}\right)=0.063 \pm 0.004$ (stat) \pm 0.001 (syst). The result for the proton, assuming $G_{E}^{p}=G_{M}^{p}$, is $G_{M}^{p}\left(13.48 \mathrm{GeV}^{2}\right)=0.014 \pm 0.002$ (stat) \pm 0.001 (syst), which is in agreement with earlier results. 
Electromagnetic form factors of hadrons are among the most important physical observables. They provide direct insight into the distribution of charges, currents, color, and flavor in the hadron. Form factors for spacelike momentum transfers, $Q^{2}>0$, are determined by elastic scattering of electrons from hadrons available as targets. Form factors for timelike momentum transfers, $Q^{2}<0$, are measured by annihilation $e^{+} e^{-} \leftrightarrow h^{+} h^{-}$. They lead to insight into the wave function of the hadron in terms of its partonic constituents. In this Letter we report on the first precision measurements for the timelike electromagnetic form factors of the pion, kaon, and proton, for $\left|Q^{2}\right|=13.48 \mathrm{GeV}^{2}$, by means of the reactions

$$
e^{+} e^{-} \rightarrow \pi^{+} \pi^{-}, \quad K^{+} K^{-}, \quad \text { and } \quad p \bar{p} .
$$

Measurements of the timelike form factors of pion and kaon, $F_{\pi}$ and $F_{K}$, with identified pions and kaons exist for $\left|Q^{2}\right| \leq 4.5 \mathrm{GeV}^{2}$ [1]. For larger $\left|Q^{2}\right|$ either only upper limits for $F\left(\left|Q^{2}\right|\right)$ exist or the few observed hadron pairs, not separately identified as pions or kaons, are divided between the two according to the expectations based on the vector dominance model (VDM) to obtain $F_{\pi}$ and $F_{K}$ [2].

Timelike form factors of the proton for $\left|Q^{2}\right|>6 \mathrm{GeV}^{2}$ were first measured by the Fermilab E760/E835 experiments via the reaction $p \bar{p} \rightarrow e^{+} e^{-}$[3]. According to perturbative QCD (pQCD) at large momentum transfers the timelike form factors of protons are expected to be nearly equal to the spacelike form factors, but the Fermilab measurements found the timelike form factors to be nearly twice as large. In this Letter we provide the first independent confirmation of the Fermilab observations.

Theoretical predictions of form factors based on pQCD rely on the validity of factorization for sufficiently high momentum transfers and lead to quark counting rules, which predict [4] that $F\left(\left|Q^{2}\right|\right) \propto\left|Q^{2}\right|^{1-n}$, where $n$ is the number of quarks, so that $F\left(\left|Q^{2}\right|\right) \propto \alpha_{S} /\left|Q^{2}\right|$ for mesons, and $F\left(\left|Q^{2}\right|\right) \propto \alpha_{S}^{2} /\left|Q^{4}\right|$ for baryons. pQCD also predicts [5] that the form factors for the helicity-zero mesons $m=\pi, K, \rho, \ldots$ are proportional to the squares of their decay constants so that $F_{\pi}\left(\left|Q^{2}\right|\right) / F_{K}\left(\left|Q^{2}\right|\right)=$ $f_{\pi}^{2} / f_{K}^{2}$, as $\left|Q^{2}\right| \rightarrow \infty$.

By providing tests of the above predictions, we expect to shed light on the important question of the momentum transfers which are sufficiently large to validate the use of pQCD, a question which has been in debate for a long time $[6,7]$.

The timelike form factors of the charged helicity-zero mesons are related to the differential and total cross sections for their pair production by

$$
\frac{d \sigma_{0}(s)}{d \Omega}\left(e^{+} e^{-} \rightarrow m^{+} m^{-}\right)=\frac{\alpha^{2}}{8 s} \beta_{m}^{3}\left|F_{m}(s)\right|^{2} \sin ^{2} \theta,
$$

where $m=\pi$ or $K, \alpha$ is the fine-structure constant, $\beta_{m}$ is the meson velocity in the laboratory system, $s=\left|Q^{2}\right|$ is the center-of-mass energy squared, $\left|F_{m}(s)\right|$ is the electromagnetic form factor of the meson, and $\theta$ is the laboratory angle between the meson and the positron beam.

The $e^{+} e^{-} \rightarrow p \bar{p}$ differential cross sections are related to the magnetic $\left(G_{M}^{p}\right)$ and electric $\left(G_{E}^{p}\right)$ form factors of the proton. With $\tau=4 m_{p}^{2} / s$

$$
\begin{aligned}
\frac{d \sigma_{0}(s)}{d \Omega}= & \frac{\alpha^{2}}{4 s} \beta_{p}\left[\left|G_{M}^{p}(s)\right|^{2}\left(1+\cos ^{2} \theta\right)\right. \\
& \left.+\tau\left|G_{E}^{p}(s)\right|^{2} \sin ^{2} \theta\right] .
\end{aligned}
$$

In the present measurements we do not have sufficient statistics to separate $G_{E}^{p}(s)$ and $G_{M}^{p}(s)$. We therefore analyze our data with the two assumptions, $G_{E}^{p}=G_{M}^{P}$ and $G_{E}^{p}=0$, as in Ref. [3].

The $e^{+} e^{-}$annihilation data samples used in the present measurements consist of $20.7 \mathrm{pb}^{-1}$ at $\sqrt{s}=3.671 \mathrm{GeV}$ and $2.89 \mathrm{pb}^{-1}$ at the $\psi(2 S)$ mass, $\sqrt{s}=3.686 \mathrm{GeV}$. The data were collected at the Cornell Electron Storage Ring (CESR) with the detector in the CLEO- $c$ configuration [8]. The detector is cylindrically symmetric and provides $93 \%$ coverage of solid angle for charged and neutral particle identification. The detector components important for this analysis are the wire vertex detector (ZD), the main drift chamber (DR), the ring-imaging Cherenkov detector (RICH), and the CsI crystal calorimeter (CC). They are operated within a $1.0 \mathrm{~T}$ magnetic field produced by a superconducting solenoid located directly outside of the CC. The ZD and DR detect charged particles, and the DR provides measurement of their momenta with a precision of $\sim 0.6 \%$ at $p=1 \mathrm{GeV} / c$ and ionization energy loss $(d E / d x)$. The RICH detector provides particle identification and covers $80 \%$ of the solid angle. The combination of $d E / d x$ and information from the RICH detector allows for separating different charged particle species. The CC allows precision measurements of electromagnetic shower energy and position.

A fully reconstructed event is required to have two charged particles and zero net charge. The charged particles in the $\pi^{+} \pi^{-}$final state analysis must have $|\cos \theta|<$ 0.75 and have an associated shower in the CC. The charged particles in the $K^{+} K^{-}$and $p \bar{p}$ analyses must have $|\cos \theta|<0.80$. Each charged particle is required to satisfy standard requirements for track quality and distance of closest approach to the interaction point. For the $\pi^{+} \pi^{-}$ and $p \bar{p}$ analyses, the two charged particles must have a net momentum $<100 \mathrm{MeV} / c$, and for the $K^{+} K^{-}$analysis, they must have a net momentum $<60 \mathrm{MeV} / c$.

The hadronic $e^{+} e^{-} \rightarrow h^{+} h^{-}$processes, where $h=$ $\pi, K, p$, are studied by generating signal Monte Carlo (MC) samples, using GEANT [9] to simulate the CLEO- $c$ detector. MC samples of the leptonic background processes $e^{+} e^{-} \rightarrow l^{+} l^{-}(l=e, \mu)$ are also studied. Figure 1 shows the MC distributions for two track final states which pass 


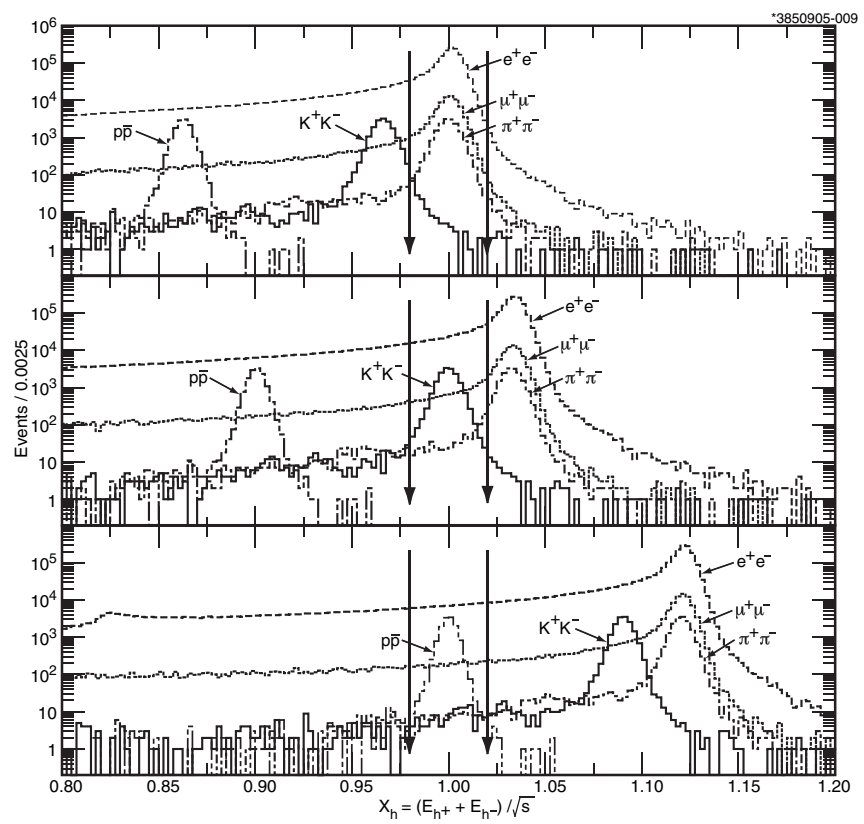

FIG. 1. MC distributions as a function of $X_{h} \equiv\left(E_{h+}+\right.$ $\left.E_{h-}\right) / \sqrt{s}$, where $h=\pi$ (top), $h=K$ (middle), and $h=p$ (bottom). The signal regions are defined as $0.98<X_{h}<1.02$ and are designated by the vertical arrows.

the $\pi^{+} \pi^{-}, K^{+} K^{-}$, and $p \bar{p}$ criteria described above. The normalized center-of-mass energy variable $X_{h}$ is defined as the sum of the energy of the two tracks (assuming the particle species of interest for each track) divided by $\sqrt{s}$. Figure 1 shows that the $e^{+} e^{-} \rightarrow K^{+} K^{-}$and $e^{+} e^{-} \rightarrow p \bar{p}$ signal regions are sufficiently displaced from the dominant $e^{+} e^{-} \rightarrow l^{+} l^{-}$background, while the $e^{+} e^{-} \rightarrow \pi^{+} \pi^{-}$signal overlaps with $e^{+} e^{-} \rightarrow l^{+} l^{-}$.

In order to suppress $l^{+} l^{-}$background events, it is first required that the accepted events have the ratio of the $\mathrm{CC}$ determined energy $E_{\mathrm{CC}}$ and the track determined momentum $p, E_{\mathrm{CC}} / p$, be less than 0.85 . For $p \bar{p}$ events this cut is applied only on the positive track; for $\pi^{+} \pi^{-}$ and $K^{+} K^{-}$it is applied on both tracks. In order to obtain a higher level of lepton rejection, signal to background optimization studies are made in terms of a likelihood variable defined by using RICH and $d E / d x$ information, $L(p, K)-L(l)=L_{\mathrm{RICH}}(p, K)-L_{\mathrm{RICH}}(l)+$ $\sigma_{d E / d x}^{2}(p, K)-\sigma_{d E / d x}^{2}(l)$. The optimum requirement is found to be $L(p, K)-L(e)<0$, and $L(p, K)-L(\mu)<$ -2 for each track.

Rejecting leptonic background in the $\pi^{+} \pi^{-}$sample requires additional measures. These are determined by studying radiative Bhabha events in the continuum $(\sqrt{s}=$ $3.671 \mathrm{GeV})$ data, by studying $\mu$ tracks from the $e^{+} e^{-} \rightarrow$ $\mu^{+} \mu^{-}$Monte Carlo sample, and by studying pions of appropriate momenta $(\sim 1.83 \mathrm{GeV} / c)$ in the existing CLEO sample of inclusive $D^{0} \rightarrow K^{-} \pi^{+}$data taken at $\sqrt{s}=$ $10.58 \mathrm{GeV}$. The optimization criteria which emerged from these studies are that pions must deposit $E_{\mathrm{CC}}>$

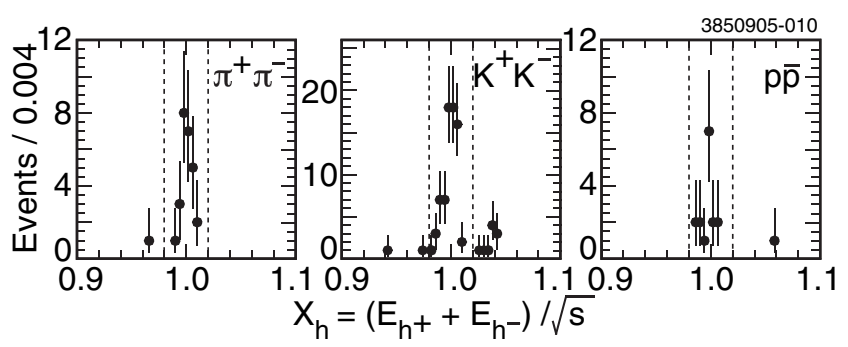

FIG. 2. Data events as a function of $X_{h}$ for $\pi^{+} \pi^{-}$(left), $K^{+} K^{-}$(middle), and $p \bar{p}$ (right) final states. The dashed lines denote the signal regions defined as $0.98<X_{h}<1.02$.

$0.42 \mathrm{GeV}$ and must have $L(\pi)-L(e)<0$ and $L(K)-$ $L(\pi)>0$.

Figure 2 shows the distribution of the events which meet all the above selection criteria as a function of $X_{h}$. The signal region is defined as $0.98<X_{h}<1.02$ as bounded by the dashed lines. The observed counts, the estimated lepton contamination counts, the counts contributed by the tail of the $\psi(2 S)$ resonance, and the net signal counts are listed in Table I.

The net signal counts $N$ are related to the Born cross sections as $\sigma_{0}\left(e^{+} e^{-} \rightarrow h^{+} h^{-}\right)=N /[\epsilon \mathcal{L}(1+\delta)]$, where $\epsilon$ is the detection efficiency, $\mathcal{L}$ is the total integrated luminosity, and $(1+\delta)$ is the radiative correction factor associated with $h^{+} h^{-}$production from $e^{+} e^{-}$annihilations. The proton and the kaon detection efficiencies, $\epsilon_{p}=$ $0.657 \pm 0.003$, and $\epsilon_{K}=0.743 \pm 0.003$, are determined from the signal MC samples. The pion detection efficiency, $\epsilon_{\pi}=0.166 \pm 0.002$, is determined by signal MC sample and the $D^{0} \rightarrow K^{-} \pi^{+}$data. The signal MC samples are generated with angular distributions according to Eqs. (2) and (3). The initial state radiation corrections are determined using the method of Bonneau and Martin [10] with the addition of $\mu$ and $\tau$ pair loops to the vacuum polarization term. For the final states $\pi^{+} \pi^{-}, K^{+} K^{-}$, and $p \bar{p}$, the $(1+\delta)$ correction factors are $0.832,0.810$, and 0.860 , respectively.

The resulting cross sections are listed in Table I. Various sources of systematic uncertainties in the cross sections have been studied. These are listed in Table II. Their sum in quadrature is $14.6 \%$ for pions, $4.4 \%$ for kaons, and $8.9 \%$ for protons. Integrating Eqs. (2) and (3) leads us to our final results for the form factors as listed in Table I [11].

The $2.89 \mathrm{pb}^{-1}$ of data taken at $\sqrt{s}=3.686 \mathrm{GeV}$, or the $\psi(2 S)$ resonance, were used in the form factor analysis to evaluate the contribution that the resonance makes to the form factor data at $\sqrt{s}=3.671 \mathrm{GeV}$. For this purpose the $\psi(2 S)$ data were analyzed in exactly the same way as the form factor data. Our yields in the $\psi(2 S)$ data sample, which we use to estimate the background from the $\psi(2 S)$ tail feeding into the continuum sample, is consistent with the expectation based on the branching fractions [12] for $p$ and $K$, but lower for $\pi$. 
TABLE I. Summary of form factor results. The first errors are statistical only. The second errors in cross sections and form factors are systematic errors as discussed in the text. The form factor results for protons correspond to the assumption $G_{E}^{p}=G_{M}^{P}$. Results for the assumption $G_{E}^{p}=0$ are $\sim 9 \%$ larger.

\begin{tabular}{lccc}
\hline \hline & Pion & Kaon & Proton \\
\hline No. of observed events & $26.0 \pm 5.1$ & $72.0 \pm 8.5$ & $16.0 \pm 4.8$ \\
Lepton contribution & $\sim 0.1$ & $0.6 \pm 0.2$ & $<0.1$ \\
$\psi(2 S)$ contribution & $<0.1$ & $0.6 \pm 0.1$ & $1.9 \pm 0.2$ \\
No. of net signal events & $25.9 \pm 5.1$ & $70.9 \pm 8.5$ & $14.1 \pm 4.8$ \\
$\sigma\left(e^{+} e^{-} \rightarrow h^{+} h^{-}\right)$, pb & $9.0 \pm 1.8 \pm 1.3$ & $5.7 \pm 0.7 \pm 0.3$ & $1.2 \pm 0.4 \pm 0.1$ \\
$F_{h}\left(\left|Q^{2}\right|\right)$ & $F_{\pi}=0.075 \pm 0.008 \pm 0.005$ & $F_{K}=0.063 \pm 0.004 \pm 0.001$ & $G_{M}=0.014 \pm 0.002 \pm 0.001$ \\
$Q^{n} F_{h}\left(\left|Q^{2}\right|\right), \mathrm{GeV}^{2}$ & $\left|Q^{2}\right| F_{\pi}=1.01 \pm 0.11 \pm 0.07$ & $\left|Q^{2}\right| F_{K}=0.85 \pm 0.05 \pm 0.02$ & $\left|Q^{4}\right| G_{M}=2.53 \pm 0.36 \pm 0.11$ \\
\hline \hline
\end{tabular}

Our results for timelike form factors are displayed in Fig. 3 as $\left|Q^{2}\right| F_{\pi},\left|Q^{2}\right| F_{K}$, and $\left|Q^{4}\right| G_{M}^{p} / \mu_{p}$, together with the existing world data for the same.

Our precision result for the pion, $\left|Q^{2}\right| F_{\pi}\left(13.48 \mathrm{GeV}^{2}\right)=1.01 \pm 0.11 \pm 0.07 \mathrm{GeV}^{2}$, is the first such directly measured result. It provides empirical validity for $\left|Q^{2}\right| F_{\pi}\left(9.6 \mathrm{GeV}^{2}\right)=(0.94 \pm 0.06) \mathrm{GeV}^{2}$ obtained by interpreting $\Gamma\left(J / \psi \rightarrow \pi^{+} \pi^{-}\right) / \Gamma\left(J / \psi \rightarrow e^{+} e^{-}\right)$ as a measure of the pion form factor [13]. Together, the two appear to support the pQCD prediction of the $\alpha_{S} /\left|Q^{2}\right|$ variation of the form factor at large $\left|Q^{2}\right|$. Bebek et al. [14] have reported $\left|Q^{2}\right| F_{\pi}\left(9.77 \mathrm{GeV}^{2}\right)=0.69 \pm$ $0.19 \mathrm{GeV}^{2}$ for the spacelike form factor. Within errors this is consistent with being nearly a factor of 2 smaller than the timelike form factors for $Q^{2}>9 \mathrm{GeV}^{2}$, as found for protons.

Our measurement of the kaon form factor stands alone at present. The asymptotic pQCD prediction is [5] $F_{\pi, K}\left(\left|Q^{2}\right|\right)=8 \pi \alpha_{s} f_{\pi^{+}, K^{+}}^{2} /\left|Q^{2}\right|$. For $\alpha_{s}=0.3, f_{\pi^{+}}=$ $130.7 \pm 0.4 \mathrm{MeV}$, and $f_{K^{+}}=159.8 \pm 1.5 \mathrm{MeV}$ [12], this leads to $F_{\pi}\left(13.48 \mathrm{GeV}^{2}\right)=0.010$ and $F_{K}\left(13.48 \mathrm{GeV}^{2}\right)=0.014$, which are factors of $\sim 8$ and $\sim 4$ smaller than our results, respectively. The $\alpha_{s}$ and $Q^{2}$ independent $\mathrm{pQCD}$ prediction $F_{\pi}\left(\left|Q^{2}\right|\right) / F_{K}\left(\left|Q^{2}\right|\right)=$

TABLE II. Sources of systematic uncertainty for the $e^{+} e^{-} \rightarrow$ $\pi^{+} \pi^{-}, e^{+} e^{-} \rightarrow K^{+} K^{-}$, and $e^{+} e^{-} \rightarrow p \bar{p}$ cross sections.

\begin{tabular}{lccc}
\hline \hline Source & $\pi^{+} \pi^{-}(\%)$ & $\mathrm{K}^{+} K^{-}(\%)$ & $p \bar{p}(\%)$ \\
\hline Trigger & 1.0 & 1.0 & 1.0 \\
Tracking & $2 \times 1.0$ & $2 \times 1.0$ & $2 \times 1.0$ \\
$X_{h}$ signal region & 4.1 & 0.5 & 3.7 \\
Net momentum & 4.8 & 2.6 & 6.9 \\
$d E / d x+$ RICH PID & $2 \times 2.7$ & $2 \times 1.2$ & $2 \times 1.6$ \\
$E_{\mathrm{CC}}$ & 10.7 & $\ldots$ & $\ldots$ \\
$\epsilon_{\pi}\left(E_{\mathrm{CC}}\right)$ & $2 \times 2.3$ & $\ldots$ & $\ldots$ \\
MC statistics & 1.3 & 0.4 & 0.5 \\
$\psi(2 S)$ Contamination & 0.08 & 0.1 & 1.0 \\
Leptonic background & 0.05 & 0.3 & 0.0 \\
Radiative correction & 0.2 & 0.2 & 0.2 \\
Luminosity, $\mathcal{L}$ & 1.0 & 1.0 & 1.0 \\
Total (in quadrature $)$ & 14.6 & 4.4 & 8.9 \\
\hline \hline
\end{tabular}

$f_{\pi}^{2} / f_{K}^{2}=0.67 \pm 0.01$ is also in disagreement with our result $F_{\pi}\left(13.48 \mathrm{GeV}^{2}\right) / F_{K}\left(13.48 \mathrm{GeV}^{2}\right)=1.19 \pm 0.17$. Bakulev et al. [15] have estimated soft contributions to the pion form factor in the framework of QCD sum rules.

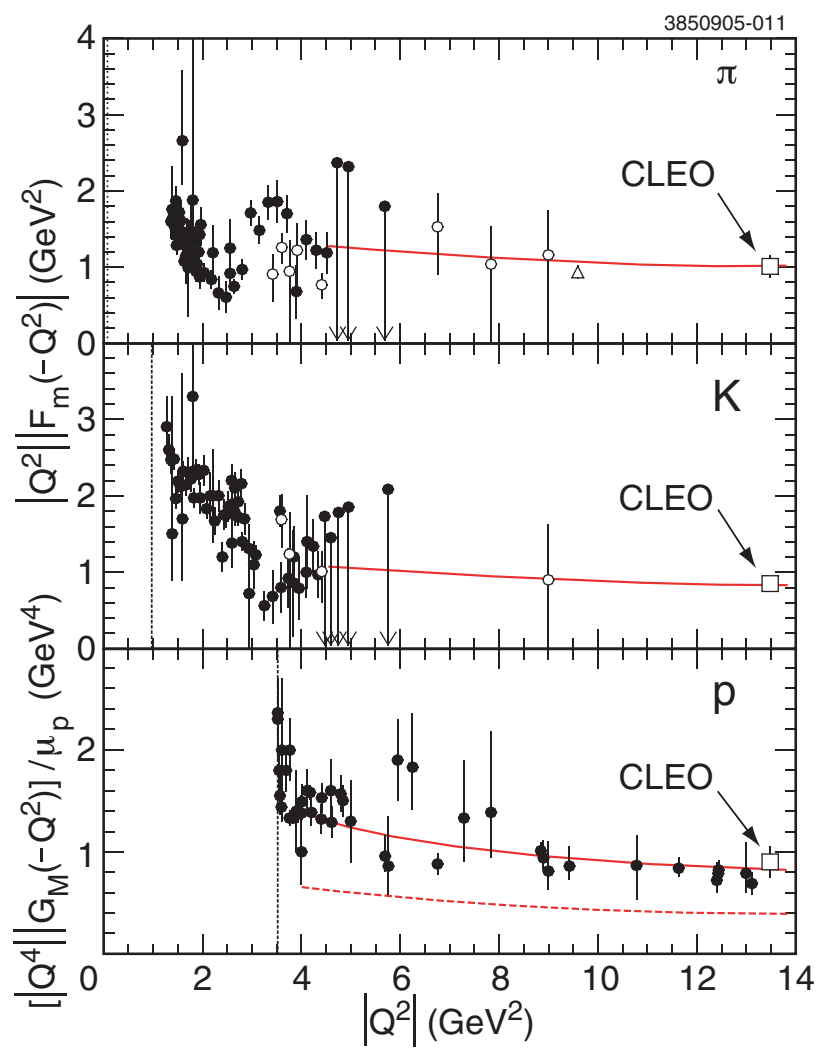

FIG. 3 (color online). Compilation of the existing experimental data for the pion (top), kaon (middle), and proton (bottom) form factors with timelike momentum transfer. Top and middle: the solid points are from identified $\pi^{ \pm}$and $K^{ \pm}$[1]. The open points are from unidentified $h^{ \pm}$, divided into $\pi^{ \pm}$and $K^{ \pm}$ according to VDM expectations. For pions (top) the open triangle denotes the value obtained at $M^{2}(J / \psi)$ in Ref. [13]. Bottom: the solid points for proton form factors are from the measurements and compilation of Ref. [3]. The arbitrarily normalized solid curves show the variation of $\alpha_{S}\left(\left|Q^{2}\right|\right)$ (top and middle) and $\alpha_{S}^{2}\left(\left|Q^{2}\right|\right)$ (bottom), as determined for four flavors and $\Lambda=0.322 \mathrm{GeV}$. The dashed curve in the bottom plot shows $\alpha_{S}^{2}\left(\left|Q^{2}\right|\right)$ fit to the spacelike form factors of the proton. 
Their formulation leads to $F_{\pi}\left(13.48 \mathrm{GeV}^{2}\right)=0.010$ and $F_{\pi}\left(\left|Q^{2}\right|\right) / F_{K}\left(\left|Q^{2}\right|\right)=0.51$; i.e., the discrepancy between our experimental results and the theoretical predictions remains. We note that this behavior is in contrast to the good agreement between the measured $\pi^{0} \gamma$ transition form factor and the pQCD prediction for the same [16].

Our result for $G_{M}^{p}\left(13.48 \mathrm{GeV}^{2}\right)$ is in excellent agreement with the results of the Fermilab E760/E835 experiments in which the reverse reaction $p \bar{p} \rightarrow e^{+} e^{-}$was measured [3]. Our results provide the first independent confirmation of the Fermilab results.

We gratefully acknowledge the effort of the CESR staff in providing us with excellent luminosity and running conditions. This work was supported by the National Science Foundation and by the U.S. Department of Energy.

[1] For a compilation of pion and kaon form factor data, see M. R. Whalley, J. Phys. G 29, A1 (2003).

[2] M. Bernardini et al., Phys. Lett. 46B, 261 (1973); D. Bollini et al., Lett. Nuovo Cimento 14, 418 (1975); D. Bisello et al., Phys. Lett. B 220, 321 (1989); D. Bisello et al., Z. Phys. C 39, 13 (1988).

[3] T. A. Armstrong et al. (E760 Collaboration), Phys. Rev. Lett. 70, 1212 (1993); M. Ambrogiani et al. (E835 Collaboration), Phys. Rev. D 60, 032002 (1999); M. Andreotti et al. (E835 Collaboration), Phys. Lett. B 559, 20 (2003), and references therein.

[4] S. J. Brodsky and G. R. Farrar, Phys. Rev. Lett. 31, 1153 (1973); Phys. Rev. D 11, 1309 (1975).

[5] G. R. Farrar and D. R. Jackson, Phys. Rev. Lett. 43, 246 (1979); S. J. Brodsky and G. P. Lepage, Phys. Lett. 87B,
359 (1979); V. Efremov and A. V. Radyushkin, Phys. Lett. 94B, 245 (1980).

[6] G.P. Lepage and S. J. Brodsky, Phys. Rev. D 22, 2157 (1980).

[7] N. Isgur and C. H. Llewellyn Smith, Phys. Rev. Lett. 52, 1080 (1984); Phys. Lett. B 217, 535 (1989); Nucl. Phys. B317, 526 (1989).

[8] Y. Kubota et al., Nucl. Instrum. Methods Phys. Res., Sect. A 320, 66 (1992); R. A. Briere et al. (CLEO-c/CESR-c Taskforces \& CLEO- $c$ Collaboration), Cornell University LEPP Report No. CLNS 01/1742, 2001 (unpublished); D. Peterson et al., Nucl. Instrum. Methods Phys. Res., Sect. A 478, 142 (2002); M. Artuso et al., Nucl. Instrum. Methods Phys. Res., Sect. A 554, 147 (2005).

[9] R. Brun et al., CERN Long Writeup W5013, 1994 (unpublished).

[10] G. Bonneau and F. Martin, Nucl. Phys. B27, 381 (1971).

[11] The present form factor analysis has been done without taking into account possible interference between continuum and $\psi(2 S)$ cross sections. For the extremes of completely constructive and completely destructive interference it is estimated that the form factors for $\pi, K$, and $p$ change by $\pm 3.5 \%, \pm 4.5 \%$, and $\pm 5.9 \%$, respectively.

[12] Review of Particle Properties, S. Eidelman et al., Phys. Lett. B 592, 1 (2004).

[13] R. Kahler and J. Milana, Phys. Rev. D 47, R3690 (1993); J. Milana, S. Nussinov, and M. G. Olsson, Phys. Rev. Lett. 71, 2533 (1993).

[14] C. J. Bebek et al., Phys. Rev. D 17, 1693 (1978).

[15] A. P. Bakulev, A. V. Radyushkin, and N. G. Stefanis, Phys. Rev. D 62, 113001 (2000).

[16] J. Gronberg et al. (CLEO Collaboration), Phys. Rev. D 57, 33 (1998). 